\title{
Cooperative Communication in Clustered Wireless Sensor Network Based on LDPC Codes
}

\author{
Yuling Zhang ${ }^{1, \mathrm{a}}$, Wenwei $\mathrm{He}^{2, \mathrm{~b}}$ \\ ${ }^{1}$ School of Information and Electrical Engineering, Ludong University, Yantai, Shandong, 264025, P. \\ R. China \\ ${ }^{2}$ School of Information and Electrical Engineering, Ludong University, Yantai, Shandong, 264025, P. \\ R. China \\ azhang-yuling@hotmail.com, 'bww19@163.com
}

Keywords: Cooperative transmission; wireless sensor network, LDPC codes.

\begin{abstract}
In this paper, considering a clustered wireless sensor network, we propose a cooperative transmission scheme based on virtual multiple antennas combining with advanced channel coding (such as low density parity check codes), and give the system performance under different conditions through simulation. The whole transmission can be divided into two stages: at the first stage, cluster head broadcasts the information and other sensors listen, which we call it intracluster broadcasting phase, at the second stage, sensors that can correctly decode the received information participate in the cooperative transmission, which we call it intercluster transmission phase. Then we minimize the overall energy consumption by adjusting the power allocation between these two transmission phase. Compared with the research so far, we apply advanced channel coding at each sensor node, which makes the system performance much better.
\end{abstract}

\section{Introduction}

In recent years, wireless sensor networks (WSN) are widely used in environmental monitoring, healthcare assistance, military applications and so on, which gain a lot of attention of researchers. Wireless sensor networks are typically energy constrained networks, sensor nodes are powered by batteries which are difficult to replace. Consequently, minimizing the energy consumption and extending the lifetime of whole network are very important issues for wireless sensor networks[1,2].

Researchers in paper [3] presented an efficient data gathering scheme that guarantees the Quality of Service, paper [4] introduced a cross-layer operation model that can improve the energy consumption and system throughput of IEEE 802.15.4 Mobile WSNs. Zhou Zhou etc. proposed cooperative communication scheme for a clustered wireless sensor network where sensors within each cluster relay data packets to nearby clusters[5], but they didn't consider the more powerful error correcting codes, such as low-density parity check codes (LDPC). Based on the research above, we propose a cooperative transmission scheme for clustered wireless sensor network based on LDPC codes with different modulation and coding schemes.

The rest of this paper is organized as follows. In Section II, we present the system model and. How to minimize the total energy is explained in Section III. In Section IV, we get numerical results through simulations. Finally, some conclusions are drawn.

\section{System Model}

The clustered wireless sensor network model we considered is shown in fig.1, the whole information transmission process can be divided into two stages. At the first stage,cluster head broadcasts the information and other sensors listen, which we call it intracluster broadcasting phase, at the second stage, sensors that can correctly decode the received information participate in the cooperative transmission, which we call it intercluster transmission phase. And at each node, Low-density parity-check codes are employed as the forward error correcting method. 


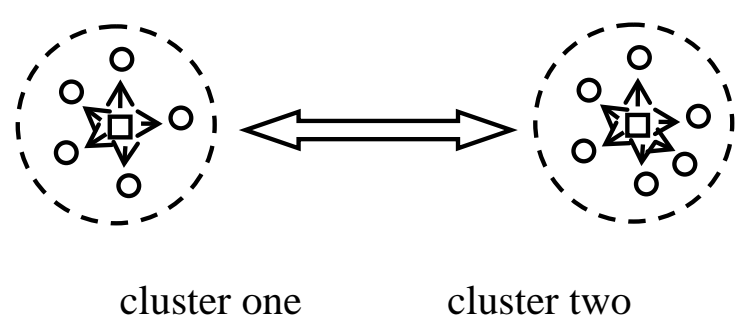

Fig.1 Clustered wireless sensor network

\section{Performance analysis}

Firstly, we analyze the performance of intracluster communication. Without generality, we assume that the cluster head (the source node) locates at the center of the cluster, and there are $\mathrm{N}$ normal sensor nodes (the receiving node) uniformly distributed in a circle with radius R1 around the cluster head. Let $r$ denote the distance between the source node and a receiving node, with the probability density function as follows

$$
f(r)=\frac{2 r}{R_{1}^{2}} \quad 0<r \leq R_{1}
$$

Under the assumption of independent Rayleigh fading, the channel coefficients are modeled as independent and identically distributed (i.i.d.) complex circular Gaussian random variables with zero mean and unit variance. The average received SNR $\bar{\gamma}_{1}$ at one node can be written as

$$
\bar{\gamma}_{1}=\frac{E_{t 1}}{N_{0} r^{2}}
$$

Where $E_{t 1}$ denotes the average transmission energy per symbol, $N_{0}$ is the one-side AWGN spectral density at the receiver, The instantaneous received SNR $\gamma_{1}$ has an exponential distribution as

$$
f\left(\gamma_{1}\right)=\frac{1}{\bar{\gamma}_{1}} e^{-\frac{\gamma_{1}}{\bar{\gamma}_{1}}}
$$

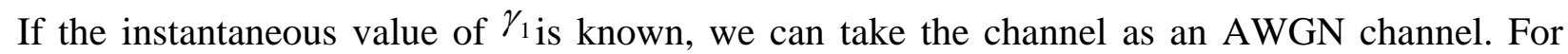
LDPC codes, an approximate expression for PER is provided as follows [6]:

$$
\operatorname{PER}_{n}\left(\gamma_{1}\right)=\left\{\begin{array}{cl}
1, & \text { if } 0<\gamma_{1}<\gamma_{c f} \\
\left(\frac{1}{1+\exp \left\{c_{n}\left(\gamma_{1}-b_{n}\right)\right\}}\right)^{a_{n}}, & \text { if } \gamma_{1} \geq \gamma_{c f}
\end{array}\right. \text {, }
$$

where $n$ depends on the modulation and coding (MCS) schemes, $\gamma_{c f}$ is the SNR cut-off value indicating that no information will be transmitted when the instantaneous SNR falls below it, $a_{n}, b_{n}$, $c_{n}$ and $\gamma_{c f}$ are parameters obtained by fitting (4) to the simulation results [7].

Given an average SNR $\bar{\gamma}_{1}$, the PER averaged over Rayleigh fading is given by

$$
\overline{\operatorname{PER}}_{1}\left(\bar{\gamma}_{1}\right)=\int_{0}^{\infty} \operatorname{PER} R_{n}\left(\gamma_{1}\right) f\left(\gamma_{1}\right) d \gamma_{1}
$$

Since $\overline{P E R}_{1}\left(\bar{\gamma}_{1}\right)$ is the function of two random variables $\bar{\gamma}_{1}$ and $E_{t 1}$, we rewrite it as $\overline{P E R}_{1}\left(E_{t 1}, r\right)$, averaging $\overline{P E R}_{1}\left(E_{t 1}, r\right)$ over the $r$ leads to

$$
\overline{\operatorname{PER}}_{1}\left(E_{t 1}\right)=\int_{0}^{R_{1}} \overline{\operatorname{PER}}_{1}\left(E_{t 1}, r\right) \frac{2 r}{R_{1}^{2}} d r
$$

Assuming that there are $M$ sensor nodes correctly decode the received packet. The probability is given by 


$$
P(M)=\left(\begin{array}{c}
N \\
M
\end{array}\right)\left[1-\overline{\operatorname{PER}}_{1}\left(E_{t 1}\right)\right]^{M}\left[\overline{\operatorname{PER}}_{1}\left(E_{t 1}\right)\right]^{N-M}
$$

The mean value of $\mathrm{M}$ is thus

$$
M_{E}=E(M)=N\left[1-\overline{P E R}_{1}\left(E_{t 1}\right)\right]
$$

At the second transmission phase, information will be transmitted between the transmitting and receiving clusters, wich we call i intercluster Cooperative Transmission.

In cluster one, the source node together with another $\mathrm{M}$ nodes (relay nodes)will jointly transmit packets using the distributed space-time block coding (STBC), so the total number of transmission nodes is $\mathrm{M} 0=\mathrm{M}+1$. Let $\mathrm{D}$ denote the distance between two clusters, and $\mathrm{D}$ is much larger than the intracluster distance, so we can approximate the transmission distances between all the transmitting nodes and the receiving node as D. then the average received SNR $\bar{\gamma}_{2}$ corresponding to each relay node is

$\bar{\gamma}_{2}=\frac{E_{t 2}}{N_{0} D^{2}}$

Where $E_{\mathrm{t} 2}$ is the transmission energy per symbol per node. The effective received SNR $\gamma_{2}$ conditional on channel realizations is

$$
\gamma_{2}=\left(\sum_{i=1}^{M_{0}}\left|h_{i}\right|^{2}\right) \bar{\gamma}_{2}
$$

Where $h_{\mathrm{i}}$ is the channel coefficient between the ith transmitting node and the receiving cluster head. We assume that the hi's are independent and identically distributed Rayleigh fading distribution. And then $\gamma_{2}$ is of a central chi-square distribution with $2 M_{0}$ degrees of freedom

$$
f\left(\gamma_{2}\right)=\frac{1}{\Gamma\left(M_{0}\right) \bar{\gamma}_{2}^{M_{0}}} \gamma_{2}^{M_{0}-1} e^{-\frac{\gamma_{2}}{\bar{\gamma}_{2}}}
$$

The average PER with $M_{0}$ transmitting nodes is then

$$
\overline{\operatorname{PER}}_{2}\left(\bar{\gamma}_{2}, M_{0}\right)=\int_{0}^{\infty} \operatorname{PER}\left(\gamma_{2}\right) f\left(\gamma_{2}\right) d \gamma_{2}
$$

Since $\overline{P E R}_{2}\left(\bar{\gamma}_{2}, M_{0}\right)$ is a function of $E_{t 2}$ and $M_{0}$, we can rewrite it as $\overline{P E R}_{2}\left(E_{t 2}, M_{0}\right)$. Combining (7),(13) and $M_{0}=M+1$, the end-to-end average PER is

$$
\begin{aligned}
& \overline{P E R}\left(E_{t 1}, E_{t 2}\right)=\sum_{M=0}^{N} P(M) \overline{P E R}_{2}\left(E_{t 2}, M+1\right) \\
& =\sum_{M=0}^{N}\left(\begin{array}{l}
N \\
M
\end{array}\right)\left[1-\overline{\operatorname{PER}}_{1}\left(E_{t 1}\right)\right]^{M} \times\left[\overline{\operatorname{PER}}_{1}\left(E_{t 1}\right)\right]^{N-M} \overline{P E R}_{2}\left(E_{t 2}, M+1\right)
\end{aligned}
$$

For simplicity, we didn't consider the circuit energy consumption, then the average total energy consumption $\bar{E}_{\text {packet }}$ for a packet is

$$
\bar{E}_{\text {packet }}=\frac{1}{b} L_{b} \times\left\{E_{t 1}+\left[N\left(1-\overline{\operatorname{PER}}_{1}\left(E_{t 1}\right)\right)+1\right] \times E_{t 2}\right\}
$$

$E_{\mathrm{t} 1}$ is the transmit energy of source node in the first phase, $E_{\mathrm{t} 2}$ is the transmit energy of source node in the second phase, $\mathrm{b}$ is the number of bits in each symbol, $L_{\mathrm{b}}$ is the number of bits in a packet.

To minimize the overall energy consumption for a packet under a certain PER requirement, the optimization problem over the transmission energy $\left(E_{\mathrm{t} 1}\right.$ and $\left.E_{\mathrm{t} 2}\right)$ can be illustrated as [5]

$\min _{E_{t 1}, E_{t 2}} \bar{E}_{\text {packet }}=\frac{1}{b} L_{b} \times\left\{E_{t 1}+\left[N\left(1-\overline{P E R}_{1}\left(E_{t 1}\right)\right)+1\right] \times E_{t 2}\right\}$

Subject to $0 \leq E_{t 1} \leq E_{\max }$ 


$$
\begin{aligned}
& 0 \leq E_{t 2} \leq E_{\max } \\
& \overline{\operatorname{PER}}\left(E_{t 1}, E_{t 2}\right) \leq \overline{\operatorname{PER}}_{0}
\end{aligned}
$$

Where $E_{\max }$ is the maximum transmitting energy per symbol allowed at each node, and $\overline{P E R}_{0}$ is the target PER specified by the users.

There are many parameters that can be adjusted to optimize the energy consumption, such as the number of nodes $\mathrm{N}$ in the cluster, cluster size R1, and cluster distance $\mathrm{D}$. But in our research, we focus on the coding rate, modulation and coding schemes, which will determine $a_{\mathrm{n}}, b_{\mathrm{n}}, c_{\mathrm{n}}$ and $\gamma_{c f}$ in the PER formula.

\section{Rate-compatible LDPC codes}

LDPC codes are block codes that exhibit near Shannon limit performance. They were first introduced by Gallager in his thesis in 1960' s [8] and rediscovered by D. J. C. Mackay after the debut of Turbo codes[9]. LDPC codes are linear block codes, which are defined by very sparse parity-check matrices $\mathrm{H}$ having dimension $\mathrm{m} * \mathrm{n}$. LDPC codes are usually represented by bipartite graphs, in which one set of nodes called the variable nodes, corresponds to the information bits of the codeword and the other set of nodes called the check nodes, corresponds to the set of parity-check constraints which define the codes. An LDPC code is called regular if every variable node participates in dv check nodes and every check node involves dc variable nodes, otherwise it is called irregular. For an irregular LDPC code, the degrees of each set of nodes are chosen according to some distribution..

RC-LDPC codes are a family of nested codes with wide range code rates generated by a low-rate LDPC code, which is the so-called mother code. A lot of work has been done to find the optimum puncturing and extending pattern, but random puncturing has been adopted in most applications. The problem of random puncturing is that the receiver cannot get the puncturing pattern easily. Therefore, it is difficult to put random puncturing into practice. Here, we proposed a simple puncturing method that is easy to implement and can get as good performance as random puncturing.

We construct the mother code by using the progressive edge growth (PEG) method[10], which has been proven to be able to produce the best LDPC codes with moderate code length and can generate a weight-increasing parity-check (WIPC) matrix. We employ LDPC codes with rate $1 / 2(1008,504)$ in our simulations. The variable node degree distribution of irregular LDPC codes is as follows

$$
\lambda(x)=\sum_{i} \lambda_{i} x^{i}=0.47532 x^{2}+0.27953 x^{3}+0.03486 x^{4}+0.10889 x^{5}+0.10138 x^{15} .
$$

Then, the bits with degree 2 are all located in the right of the parity check matrix, corresponding to the parity check bits. When constructing RC-LDPC codes, puncturing those bits with lower degree can have less impact on the configuration of the mother code. So, for a given rate, we implement a continuous puncturing from those bits with the lowest degree.

\section{Numerical result}

In this section, we compare the performance of the proposed system under different modulation and coding schemes. The parameters are set as follows: $R_{1}=20 \mathrm{~m}, D=200 \mathrm{~m}, \overline{P E R}_{0}=0.01, N=10$, $N_{0}=10^{-10} \mathrm{~W} / \mathrm{Hz}$, the parameters $a_{n}, b_{n}, c_{n}$ and $\gamma_{p n}$ for PER calculation are shown in table1 [7].

Through computer simulation, we get the Overall energy consumption per packet versus $\mathrm{E}_{\mathrm{t} 1}$ under different MCS, as we can see from fig.2, the optimal $E_{t 1}$ for different MCSs are different,but very close. 
Table 1. Parameters of MCSs

\begin{tabular}{|c|c|c|c|c|}
\hline & MCS1 & MCS2 & MCS3 & MCS4 \\
\hline Modulation & BPSK & QPSK & QPSK & 16QAM \\
\hline Coding rate & $1 / 2$ & $1 / 2$ & $3 / 4$ & $3 / 4$ \\
\hline $\begin{array}{c}R_{n} \\
\text { (bits/sym) }\end{array}$ & 0.50 & 1.00 & 1.50 & 3.00 \\
\hline$a_{n}$ & 2.0711 & 2.4654 & 1.3988 & 1.2032 \\
\hline$b_{n}$ & -1.9453 & 1.1845 & 4.3105 & 10.334 \\
\hline$c_{n}$ & 3.9263 & 3.0263 & 2.9004 & 3.0533 \\
\hline$\gamma_{c f}(\mathrm{~dB})$ & -3.3017 & -0.63305 & 2.61 & 8.7682 \\
\hline
\end{tabular}

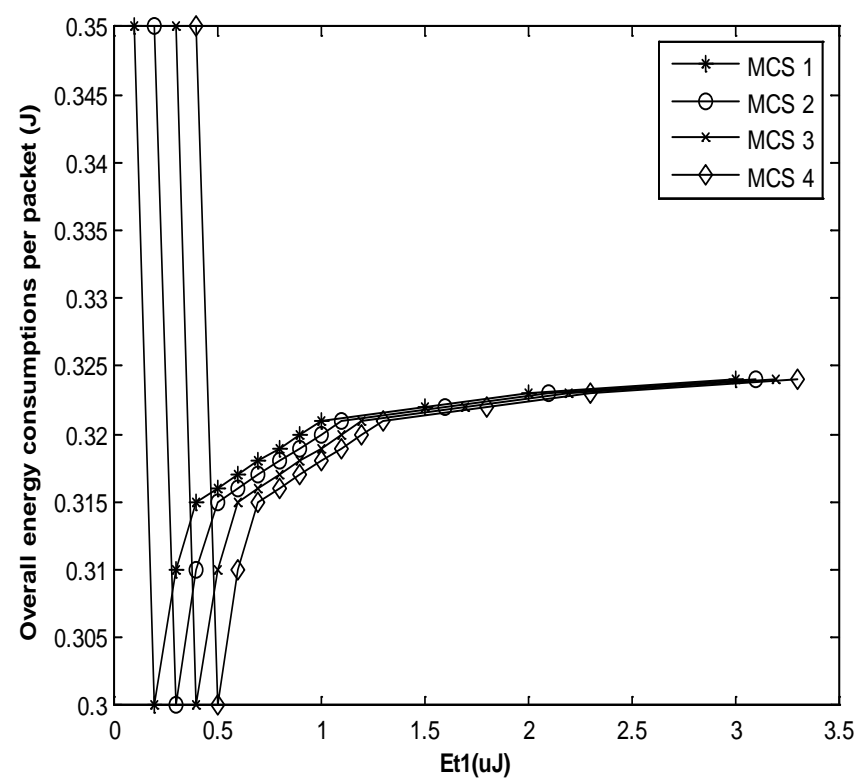

Fig.2 Overall energy consumption per packet versus $\mathrm{E}_{\mathrm{t} 1}$ under different MCS

\section{Conclusion}

In this paper, we proposed a framework of cooperative transmission schemes in clustered wireless sensor network, the powerful error correcting code LDPC codes are employed by each sensor nodes, through simulation, we give the Overall energy consumption per packet versus $E_{t 1}$ under different MCS.

\section{Reference}

[1] K. Suto, H. Nishiyama, N. Kato, Chih-Wei Huang. An Energy-Efficient and Delay-Aware Wireless Computing System for Industrial Wireless Sensor Networks. IEEE Access, 2015, 3:1026 1035.

[2] D. Kumar. Performance analysis of energy efficient clustering protocols for maximizing lifetime of wireless sensor networks. IET Wireless Sensor Systems, 2014, 4(1): 9 - 16.

[3] Jun Long, Mianxiong Dong, K. Ota etc. Reliability guaranteed efficient data gathering in wireless sensor networks. IEEE Access. 2015, 3: 430 - 444. 
[4] M. Al-Jemeli, F.A. Hussin. An Energy Efficient Cross-Layer Network Operation Model for IEEE 802.15.4-Based Mobile Wireless Sensor Networks. IEEE Sensors Journal, 2015, 15(2): 684 - 692.

[5] Zhong Zhou, Shengli Zhou, Shuguang Cui and Jun-Hong Cui. Energy-Efficient Cooperative Communication in a clustered Wireless Sensor Network. IEEE Transactions on vehicular technology, November 2008, 57(6): 3618-3628.

[6] D. J. C. MacKay and C. P. Hesketh. Performance of low density parity check codes as a function of actual and assumed noise levels. Electronic Notes in Theoretical Computer Science 74 (2003). [7] Yuling Zhang, Dongfegn Yuan, Chengxiang Wang, Cross-Layer Design Based on RC-LDPC Codes in MIMO Channels with Estimation Errors Elnteffititional Journal of Electronics and Communications, 2008; 62(9): 659-665.

[8] R. G. Gallager, Low-Density Parity-Check Codes, IRE Transactions on Information Theory, Jan. 1962.

[9]D. J. C. MacKay and R. M. Neal. Near Shannon limit performance of low density parity check codes. Electronic Letters 1996; 32 (18): 1645 - 1646.

[10]X. Hu, E. Eleftheriou, and D. M. Arnold. Regular and irregular progressive edge growth tanner graphs. IEEE Transactions on Information Theory 2005; 51 (1): 386 - 398.

\section{Acknowledgment}

This work is supported by National Scientific Foundation of China (No. 61273152, No.61471185) and Scientific Foundation of Shandong Province (No. ZR2013FL007). 